\title{
Test of the Anderson-Stuart model and correlation between free volume and the 'universal' conductivity in sodium silicate glasses
}

\author{
Marcio Luis Ferreira Nascimento
}

Received: 6 April 2006/ Accepted: 1 June 2006/Published online: 12 February 2007

(C) Springer Science+Business Media, LLC 2007

\begin{abstract}
Experimental ionic conductivity $\sigma$ and activation energy $E_{A}$ data in the binary sodium silicate system are reviewed. Analysis and brief discussion based on 48 glasses in a wide compositional range (between 4 and $45 \mathrm{Na}_{2} \mathrm{O} \mathrm{mol} \%$ ) are presented. Emphasis is placed on the application of the Anderson-Stuart model to describe the variation of activation energy $E_{A}$ with sodium concentration. In this analysis were considered experimental parameters such as shear modulus $G$ and relative dielectric permittivity $\varepsilon$, also in wide compositional range. A 'universal' finding is obtained using $\log _{10} \sigma$ vs. $E_{A} / k_{B} T$ in 47 of the 48 glasses investigated, where $E_{A}$ is the activation energy for conduction, $k_{B}$ is the Boltzmann constant and $T$ is the absolute temperature. Using conductivity and molar volume from density data, both measured at $20{ }^{\circ} \mathrm{C}$ in the same glasses, it was found a remarkable common cubic scaling relation between conductivity enhancement of the free volume due to increase in alkali content. The drastic drop in conductivity by 16 orders of magnitude for so many ionconducting binary sodium silicate glasses is then caused by structure and ion content. The effects of shear modulus, relative dielectric permittivity and free volume are taken into account, as also the problem of phase separation. In particular, it is suggested that the glass network expansion, which is related to the
\end{abstract}

M. L. F. Nascimento $(\bowtie)$

Vitreous Materials Laboratory, Department of Materials Engineering, Federal University of Sao Carlos,

Rod. Washington Luiz, km 235, Sao Carlos, SP 13565-905, Brazil

e-mail:pmlfn@iris.ufscar.br available free volume, is a parameter that could partially explain the increase in ionic conductivity for this binary system.

\section{Introduction}

High room temperature ionic conductivity in solid materials is technologically interesting for various solid state electrochemical devices such as batteries, sensors and 'smart windows'. It is well known that the ionic conductivity increases rapidly when a network glass former (for instance $\mathrm{SiO}_{2}$ ) is modified by the addition of a metal alkali such as $\mathrm{Na}_{2} \mathrm{O}$. Despite considerable experimental and theoretical attempts, there is currently no consensus regarding the diffusion mechanism involved [1], even in simple systems. Several models have been proposed, and they range from thermodynamic models based on liquid electrolytes such as the weak electrolyte [2], to models based on solid state concepts such as the jump diffusion [3], the strong electrolyte [4], and the microscopic dynamic structure [5].

Various models have been proposed for estimating the activation energy in alkali glasses. Particularly, a model suggested by Anderson and Stuart (A-S) [4] is considered to be the most directly related to physically meaning parameters, such as ionic radii, relative dielectric permittivity and elastic modulus. However, not many experimental data were available at the time the A-S theory was formulated. Moreover, no analysis has been performed for the sodium silicate system considering so many experimental data [4], and up to 
now only Hakim and Uhlmann [6] have proposed modifications on the A-S model, simply considering binary alkali silicate glasses.

The present paper reports data on ionic conductivities and activation energies of glasses in the $\mathrm{Na}_{2} \mathrm{O}-$ $\mathrm{SiO}_{2}$ system, with the purpose of correlating activation energy with composition using experimental parameters data such as shear modulus $G$ and relative dielectric permittivity $\varepsilon$. Also, a 'universal' finding is pursuit using $\log _{10} \sigma$ vs. $E_{A} / k_{B} T$. Selected experimental molar volume calculated from measured density data were used to calculate the free volume in an attempt to evaluate proposals concerning the role of an open structure for ionic conductivity. We test a general relation between the ionic conductivity enhancement and the expansion of the network forming unities, which shows that the alkali-induced volume expansion of the glass network could partially explain ionic conductivity, and that is related to the shear modulus. Dielectric permittivity is also taken into account.

\section{Experimental}

Glass samples of composition $x \mathrm{Na}_{2} \mathrm{O} \cdot(1-x) \mathrm{SiO}_{2}$ with $x=30,35,40$ and $45 \mathrm{~mol} \%$ were prepared by melting (at $1200-1400{ }^{\circ} \mathrm{C}$ for $2-4 \mathrm{~h}$ in air) $\mathrm{SiO}_{2}$ and $\mathrm{Na}_{2} \mathrm{CO}_{3}$ in a $\mathrm{Pt}-10 \% \mathrm{Rh}$ crucible. All the samples obtained were examined by optical microscopy (Jenapol, Carl Zeiss) and found to be free of visible strains, cords, bubbles and/or inclusions. The chemical composition in some samples analyzed with ICP-AES (Inductively Coupled Plasma-Atomic Emission Spectrometry) indicated a deviation from batch composition of less than $1 \mathrm{~mol} \%$. The glass transition temperature $\left(T_{g}\right)$ was determined in air with a DSC (Differential Scanning Calorimetry, Netzsch 404).

Specimens $10 \times 10 \times 1 \mathrm{~mm}^{3}$ were ground by hand on a metal plate with a slurry of water (or kerosene for higher-alkali concentration specimens), and 600-8001000 mesh SiC powder, followed by polishing with water (or kerosene) and alumina powder between 2 and $5 \mu \mathrm{m}$, from Buehler. Silver electrodes were applied by coating the two largest planes of each specimen with silver paint.

$D C$ conductivity measurements were carried out at $20-400{ }^{\circ} \mathrm{C}$ in air with a two-electrode $D C$ method. A pair of loaded-spring nickel rods was placed in a vertical furnace tube to assure good electrical contact between sample and electrode, and with windings running in opposite directions to eliminate electromagnetic fields due to heating current. Current measurements were done using a Keithley 610C electrometer $\left(10^{-1}-10^{-11}\right.$ A range), a $100 \mathrm{mV}$ Lambda tension source, and an ECIL JR temperature controller. The $A C$ measurements were carried out in another furnace (within the same temperature range), using silver paint and Pt electrodes, a HP 4192A impedance analyzer with 100$200 \mathrm{mV}$ voltage applied in the frequency $5 \mathrm{~Hz}-13 \mathrm{MHz}$ range.

Both measurements were done in air, with calibrated type $K$ thermocouples placed as near the electrodes as possible. Electrical conductivity of glasses was measured at various temperatures, from room temperature to approximately $50{ }^{\circ} \mathrm{C}$ below $T_{g}$, and all samples were kept in a dessicator between measurements. Density was measured by the Archimedes' method using a Mettler Toledo AX 204 balance coupled with a kit for density measurement (with precision up to $0.1 \mathrm{mg}$ ). These glasses were chosen because, unlike most glasses reported in the literature, data on both density and ionic conductivity were available for the same batch. More details can be found elsewhere [7].

\section{Results and discussion}

Ionic conductivity $\sigma$ in glass is a thermally activated process of mobile ions that overcome a potential barrier $E_{A}$, according to the following equation:

$\log _{10} \sigma=\log _{10} \sigma_{0}-\left(\log _{10} e\right) E_{A} / k_{B} T$

where $\sigma_{0}$ is a pre-exponential factor. Fig. 1 presents experimental data on ionic conductivity of 48 binary sodium silicate glasses ranging from $20{ }^{\circ} \mathrm{C}$ to $400{ }^{\circ} \mathrm{C}$ that follows Eq. 1 [7-25]. Conductivity data of Wrigth and Shelby [25] were estimated from experimental values of $E_{A}$ and $\sigma$ measured at $500 \mathrm{~K}$. As will be detailed below, Eq. 1 may be more usefull when one considers $\sigma=\sigma\left(E_{A}, T\right)$, leading, in fact, to a more general rule.

Many experimental works on conductivity in sodium silicate glasses have been published, basically searching for the highest conductivity values or presenting theories that apply better in certain systems, as will be cited below. However, in many works one could observe that parameters such as kind of electrode and its influence, surface preparation and conditions, heat treatments, phases involved, and others have been omitted. For common glasses at room temperature the conductivities are as low as $10^{-17} \mathrm{ohm}^{-1} \mathrm{~cm}^{-1}$, approaching the limit of the available measuring apparatus. At high alkali contents, the samples are hygroscopic, and special care on preparation procedures must be exercised. 
Fig. 1 Arrhenius plots of ionic conductivities in 48 binary sodium silicate glasses [7-25]. The temperatures measured are respectively 20 , 150,300 and $400{ }^{\circ} \mathrm{C}$.

Conductivity data of Wrigth and Shelby [25] were estimated from experimental values of $E_{A}$ and $\sigma$ measured at $500 \mathrm{~K}$

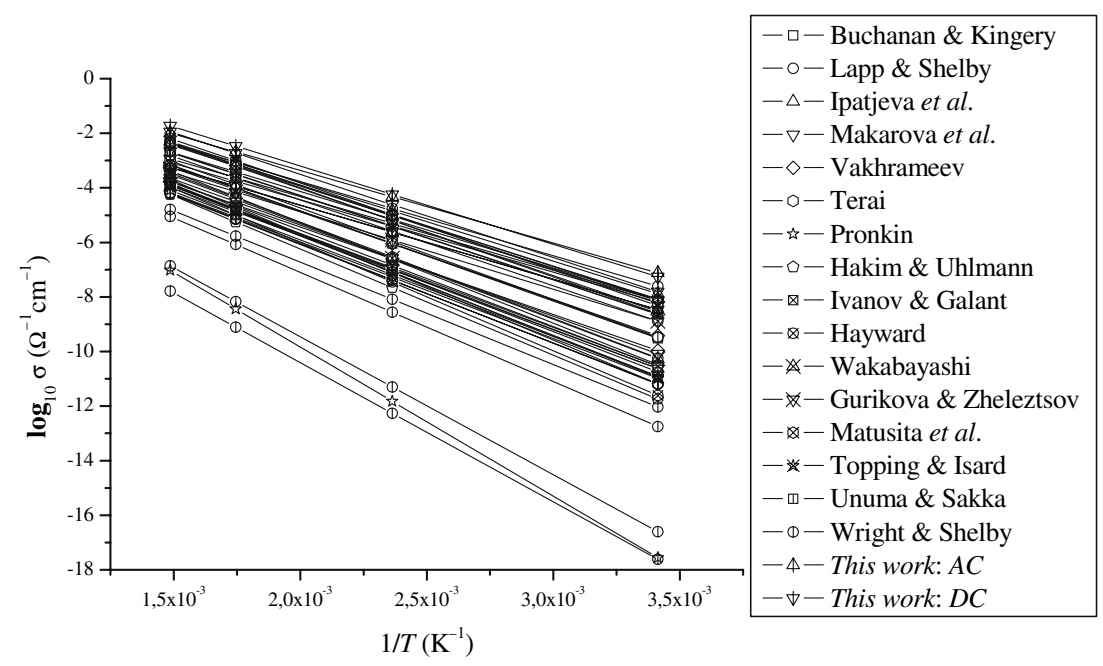

The $D C$ mode is the easiest and the most frequently used experimental procedure. Many authors [8-25] presented data on this rather simple experiment than on the more precise $A C$ mode. These data were collected and compared with our results using $D C$ and $A C$ measurements (see Fig. 1). In general, the $D C$ conductivity is slightly higher than the $A C$ conductivity considering the same system (within half an order of magnitude on $\sigma$, at a fixed temperature), mainly due to the electrode effect. This difference between $D C$ and $A C$ measurements is true only at low frequencies; at high frequencies dispersion effects should occurs. The measured $A C$ conductivity consists of intrinsic conductivity of materials along with electrode resistance and electrode-sample interface resistance. Better results frequently come from impedance $(A C)$ measurements, but good quality data could still be measured by the $D C$ mode, taking some precautions. The author did not notice any considerable difference in conductivity values using these two measurement modes. A detailed comparative analysis on $D C$ and $A C$ measurements can be found elsewhere [7].

Thus, differences observed in the activation energies, shown in Fig. 1, are likely to be associated with differences in the chemistry and/or structure of the glass samples. Figure 2 confirms this fact showing experimental molar volumes $\left(V_{m}\right)$ from density data for the same batches [7-25]. Only data from Wrigth and Shelby [25], showed in the same figure, were estimated from previous experimental values. A decrease in $V_{m}$ with alkali content can be noticed. As the structure becomes more compact with increasing alkali content, the conductivity increases. A simple question arises: how do sodium ions move in this system? Following are proposed explanations for this question.

In addition, differences in both melting and annealing procedures, sample preparation techniques and water content could affect the conduction results. In the $\mathrm{Na}_{2} \mathrm{O}-\mathrm{SiO}_{2}$ system, further differences could arise from the discussion on effects of phase separation [26].
Fig. 2 Molar volumes from densities measured at $20^{\circ} \mathrm{C}$ of 48 binary sodium silicate glasses (the same glasses are presented in Fig. 1, [7-25]). Only data from Wrigth and Shelby [25] were estimated from experimental values

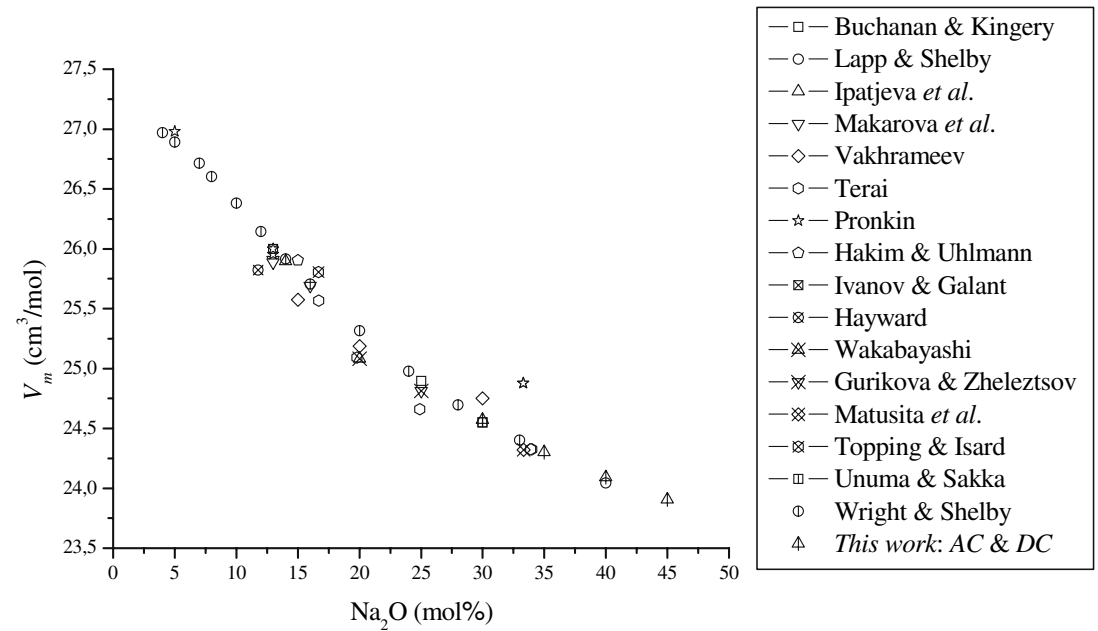


The Anderson-Stuart model

Despite few differences in the activation energies observed by different investigators, a number of common trends can be seen in those data. Perhaps most important is the decrease in activation energy with increasing the sodium concentration. It is interesting, therefore, to evaluate whether or not such behavior can be predicted from a model proposed by Anderson and Stuart [4]. In this 'classical' model, the activation energy for conductivity is divided in two parts: the electrostatic binding energy of the original site $E_{b}$, and the strain energy, $E_{s}$, required to move an ion from one site to another. $E_{b}$ describes the coulombic forces acting on the ion as it moves away from its chargecompensating site, and $E_{s}$ describes the mechanical forces acting on the ion as it dilates sufficiently the structure to allow the ion to move between sites. The basic idea is that an ion (in this case $\mathrm{Na}^{+}$) makes a simple jump from one site to another, and passes through a 'doorway' which opens as it passes through, where cation sites require only the presence of nonbridging oxygens. The A-S model follows Eq. 2:

$E_{A}=\frac{\beta z z_{0} e^{2}}{\varepsilon\left(r_{N a}+r_{O}\right)}+4 \pi G r_{D}\left(r_{N a}-r_{D}\right)^{2}$

where $z$ and $z_{0}$ are the valence of the mobile ion and of the fixed counterion-in this case sodium and oxygen, respectively; $r_{\mathrm{Na}}$ and $r_{\mathrm{O}}$ are the corresponding Pauling ionic radii for $\mathrm{Na}^{+}$and $\mathrm{O}^{2-}, e$ is the electronic charge, and $r_{\mathrm{D}}$ is the effective radius of the (un-opened) doorway.

The parameters of interest in the A-S model are the elastic modulus $(G)$, the 'Madelung' constant $(\beta)$ which depends on how far apart the ions are, and the relative dielectric permitivitty $(\varepsilon)$ which indicates the degree of charge neutralization between the ion and its nearest neighbours [4]. Martin and Angell [27] have provided a visualization of the energetics of the conduction process in an ionic conducting glass based on the A-S model. McElfresh and Howitt [28] suggested a modification on the $E_{s}$ term, where they included the jumping distance $\lambda$ as a more appropriate parameter (Eq. 3).

$E_{A}=\frac{\beta z z_{0} e^{2}}{\varepsilon\left(r_{N a}+r_{O}\right)}+4 \pi G \lambda\left(r_{N a}-r_{D}\right)^{2}$

Two considerations are proposed here: (a) One is related with the shear modulus $G$. Even considering scattering, available $G$ data decrease slightly with increasing $\mathrm{Na}_{2} \mathrm{O}$ mol\% concentration, as shown in Fig. 3 [29-38]; (b) We also consider $\beta$ as in the Anderson and Stuart model [4]:

$\beta=\frac{a-r_{N a}}{b}$

where $r_{\mathrm{Na}}$ is a value given in $\AA$; $a$ and $b$ will be defined below.

Two hypotheses were taken into account in this work: (i) $r_{\mathrm{D}}$ fitting all data, as suggested by the A-S theory, Eq. 2. (ii) $\lambda$ fitting all data, following McElfresh and Howitt's suggestion [28], Eq. 3. In this case, $r_{\mathrm{D}}$ was considered as a fitting parameter just for comparison.

The shear modulus $G$ from many authors (Fig. 3) [29-38] showed a small decrease with increasing sodium content, but there is an evident scattering. In this work we followed a linear fit close to the Appen's calculations [29], which suggested a linear decrease in
Fig. 3 Measured shear modulus $(G$, in $\mathrm{GPa})$ of the $\mathrm{Na}_{2} \mathrm{O}-\mathrm{SiO}_{2}$ system [29-38]. Fit on all data (dotted line). Note that data follow Appen's theoretical calculations tendency (full line)

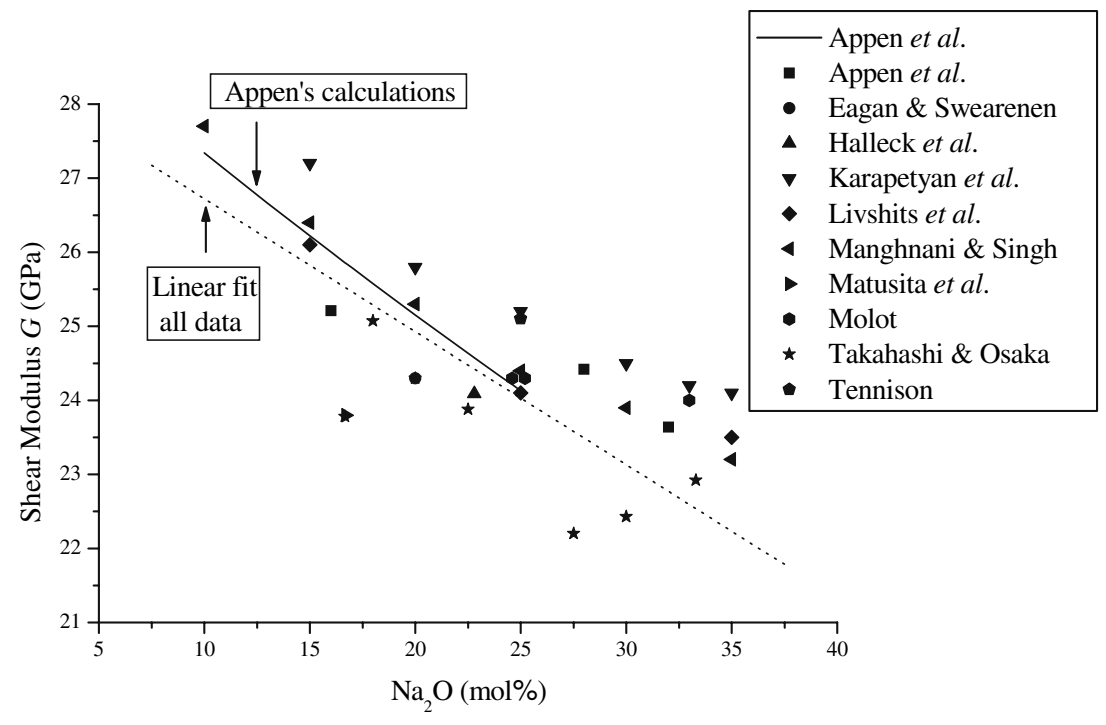


$G$ with increasing the sodium content $n$ in the sodium silicate system (Eq. 5):

$G=G_{0}-\frac{d G}{d n} n$,

where $G_{0}=(28.52 \pm 0.57) \mathrm{GPa}, n$ is the $\mathrm{Na}_{2} \mathrm{O}$ mole $\%$ composition and $d G / d n=(0.179 \pm 0.024) \mathrm{GPa} / \mathrm{mole} \%$. It is recognized that this assumption of a similitude of form may provide an inadequate description of the change in $G$ with alkali concentration, therefore it seems to provide only an approximation.

In Fig. 4, the relative dielectric permittivity $\varepsilon$ from many authors [38-43] showed also a small and monotonic increase with increasing sodium content, without evident scattering as in the shear modulus $G$, although showing two data sets. In this work, it was considered

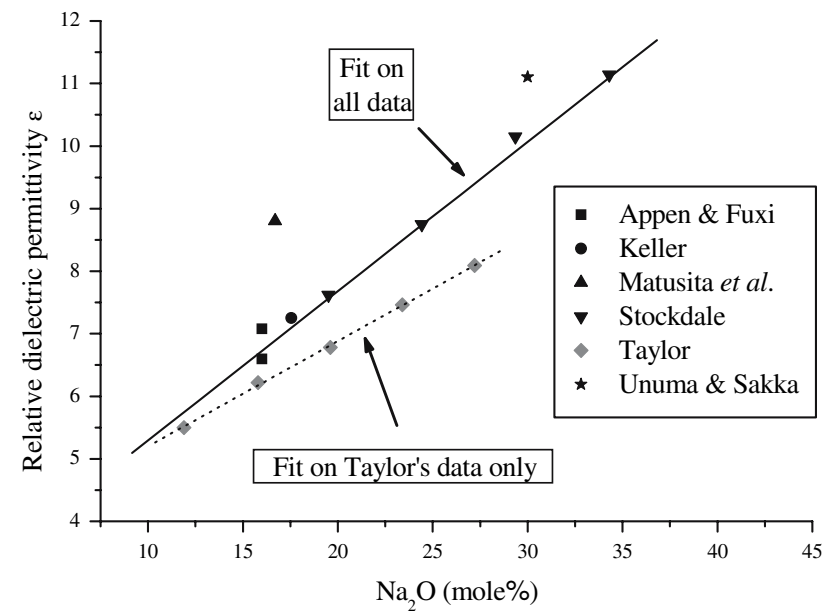

Fig. 4 Experimental relative dielectric permittivity values $(\varepsilon)$ of the $\mathrm{Na}_{2} \mathrm{O}-\mathrm{SiO}_{2}$ system [38-43] considering all data (full line) or only Taylor's data (dotted line) the fitting on all data (except Taylor's data, which used the bridging method at higher frequencies). The linear increase in $\varepsilon$ with increasing sodium content $n$ in this system follows:

$\varepsilon=\varepsilon_{0}+\frac{d \varepsilon}{d n} n$,

where $\varepsilon_{0}=2.91 \pm 0.66$ and $d \varepsilon / d n=(0.239 \pm 0.028) /$ mole.

The variation of activation energy $E_{A}$ with $\mathrm{Na}_{2} \mathrm{O}$ mole \% content in 48 glasses is shown in Fig. 5, and these data correspond to the same experimental data in Figs. 1 and 2. A careful analysis was carried out to find some possible discrepancies on the scattering. Phase separation has also been shown to play a major role in controlling the conductivity of glasses. In many cases the morphology of the glass is far more important than the bulk composition in determining this property [26]. Ionic diffusion involves mass transport over extended distances, and in principle it is particularly sensitive to changes in morphology. The sodium silicate system is known to be phase separated over an appreciable range of compositions (from nearly pure silica to about $20 \mathrm{Na}_{2} \mathrm{O} \mathrm{mol} \%$ ), and its immiscibility boundary as well as its phase separation characteristics have been determined [26].

Ionic conductivity for phase separated glasses is controlled by the connectivity of the carrier-rich phase. Modifier-rich droplets in a silica rich matrix would require the migration of sodium carrier ions through the silica rich phase for ionic conductivity. Since such phase has a very low conductivity $\sigma$ and a high activation energy $E_{A}$, the conductivity of the overall sample would be low and the activation energy would be that of the silica-rich phase. If
Fig. 5 Non-linear adjust (full line) on activation energies in $48 \mathrm{Na}_{2} \mathrm{O}-\mathrm{SiO}_{2}$ glasses [7-25] considering assumption ii. $E_{b}$ is the binding energy (dashed line), and $E_{s}$ the strain energy (dotted line)

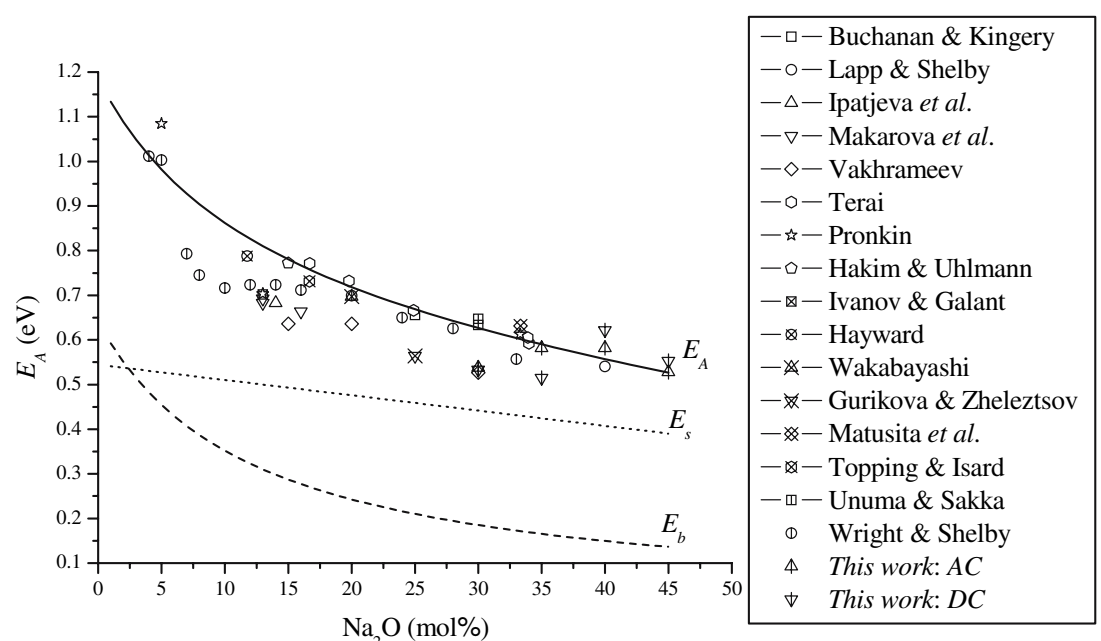


continuous migration paths for the sodium ions can be found within the modifier-rich phase, conductivity will be much greater and the activation energy $E_{A}$ will be representative of that of the modifier-rich phase.

However, the results of activation energies $E_{A}$ against $\mathrm{Na}_{2} \mathrm{O}$ mole \% content in Fig. 5 prove not to be strongly dependent on the morphology of the sample in the immiscibility sodium silicate gap. For instance, Hakim and Uhlmann [17] verified by transmission electron microscopy that compositions of 5, 20 and $25 \mathrm{Na}_{2} \mathrm{O}$ mole\% were not phase-separated in an observable scale, whereas those of 12.5 and $15 \mathrm{Na}_{2} \mathrm{O}$ mole \% content showed extensive separation. Thus, it is worth note that data in Fig. 5 include results on glasses that were noticeably phase phase-separated, not phase separated, and others with no indication.

At high alkali content samples are hygroscopic, and special precautions in the preparation and measuring procedures must be taken to ensure the absence of surface or bulk proton conduction (from water content). These processes would modify $\sigma$ conductivities, and consequently change $E_{A}$ activation energies. Fortunately, the effect of water content on conduction in sodium silicate glass has been studied in the $\mathrm{Na}_{2} \mathrm{O}$. $3 \mathrm{SiO}_{2}$ system by Takata et al. [44], who showed that the conductivity-water content is similar to the "mixedalkali" effect, and suggested a similar interaction between $\mathrm{Na}^{+}$and water $\left(\mathrm{H}^{+}\right.$or $\left.\mathrm{H}_{3} \mathrm{O}^{+}\right)$. Further details on this specific problem could be found elsewhere [45].

Besides some scattering, effects of glass composition on $E_{A}$ could be parametrized by the A-S theory. This model could even be applied in alkali silicate glasses to predict, for example, the dependence of of $E_{A}$ with $\mathrm{Na}_{2} \mathrm{O}$ content considering non-separated glass samples. Figure 5 presents data that support the discussion above.

With regard to the fitting procedure, in the first case radii values were fixed as in the $\mathrm{A}-\mathrm{S}$ model for the sodium silicate system $\left(r_{\mathrm{Na}}=0.95 \AA\right.$ and $r_{\mathrm{O}}=1.4 \AA$, full line). The fitting parameter was the doorway radius, that resulted in $r_{\mathrm{D}}=0.32 \AA$. The second assumption was a high 'doorway' radius value giving $r_{\mathrm{D}}=0.55 \AA$ and a jumping distance $\lambda=1.54 \AA$, and both were used as fitting parameters. Shear modulus $G$ and the relative dielectric permittivity $\varepsilon$ were used on both assumptions for all data (Figs. 3-4). The $\beta$ parameter varied between 0.25 and 0.15 considering assumptions $i(a=1.83 ; b=3.51)$ and $i i(a=1.39$; $b=2.89$ ), respectively. Just for comparison, the original $\beta$ value for the sodium silicate system considered by Anderson and Stuat was 0.33 (Eq. 1), but the author presume that this value obtained is inconsistent with the scaling relation presented in the next section.

The adjustment for activation energy $E_{A}$ in Fig. 5 was performed using a Levenberg-Marquardt nonlinear fitting. It is surprising that a simple theory could adjust a lot of data from several authors with different glass preparation processes in a wide composition range. With respect to the model, the analysis using shear modulus $G$ showed more influence on the A-S theory than using experimental relative dielectric permittivity $\varepsilon$, considering hyposthesis (ii).

In summary, results in Fig. 5 showed that $E_{s}$ is higher than $E_{b}$ considering the Anderson-Stuart hypothesis (ii). The same does not take place considering hypothesis (i), albeit the sum $E_{b}+E_{s}$ is similar. It is interesting to note that the $E_{b}$ dependence on the $\mathrm{Na}_{2} \mathrm{O}$ content is related to the relative dielectric permittivity $\varepsilon$, which increases with the sodium content. The main difference between hypothesis (i) and (ii) is related to the experimental shear modulus $G$.

The 'universal' conductivity and the free volume

Extensive studies have recently been made for obtaining a 'universal' equation from the glass structure standpoint. For example, Nascimento et al. [46] presented 23 and 30 binary rubidium and cesium silicate glasses, respectively, that follow a 'universal' conductivity rule. Swenson and Börjesson [47] proposed a common cubic scaling relation between $\sigma$ and the expansion volume of the network forming units in salt-doped and -undoped glasses. This fact suggested that the glass network expansion, which is related to the available free volume, is a key parameter determining the increase of the high ionic conductivity in some types of fast ion conducting glasses.

According to Adams and Swenson [48], the ion conduction should be determined by the ionic motion within an infinite pathway cluster. For various silver ion conducting glasses [49-51], it was found that the cubic root of the volume fraction $F$ of infinite pathways for a fixed valence mismatch threshold is closely related to both the absolute conductivity and the activation energy of the conduction process:

$\log _{10} \sigma T \approx \sqrt[3]{F}=\log _{10} \sigma_{0}^{\prime}-\left(\log _{10} e\right) E_{A} / k_{B} T$

where $\sigma_{0}{ }^{\prime}$ is the pre-exponential factor (in $\mathrm{K} / \Omega \cdot \mathrm{cm}$ ). The cubic root of $F$ may be thought of as proportional to the mean free path of the mobile ion [47].

Nascimento and Watanabe [52-54] have recently verified this 'universal' finding in binary silicate, borate 
Fig. 6 Modified Arrhenius plots of ionic conductivities in 48 binary sodium silicate glasses. All data correspond to that presented in Figs. 1 and 5 . The dashed line is the 'universal curve', Eq. 1, with $\sigma_{0}=50 / \Omega \mathrm{cm}$, and the dotted lines correspond to one order of magnitude higher or lower than in Eq. 1

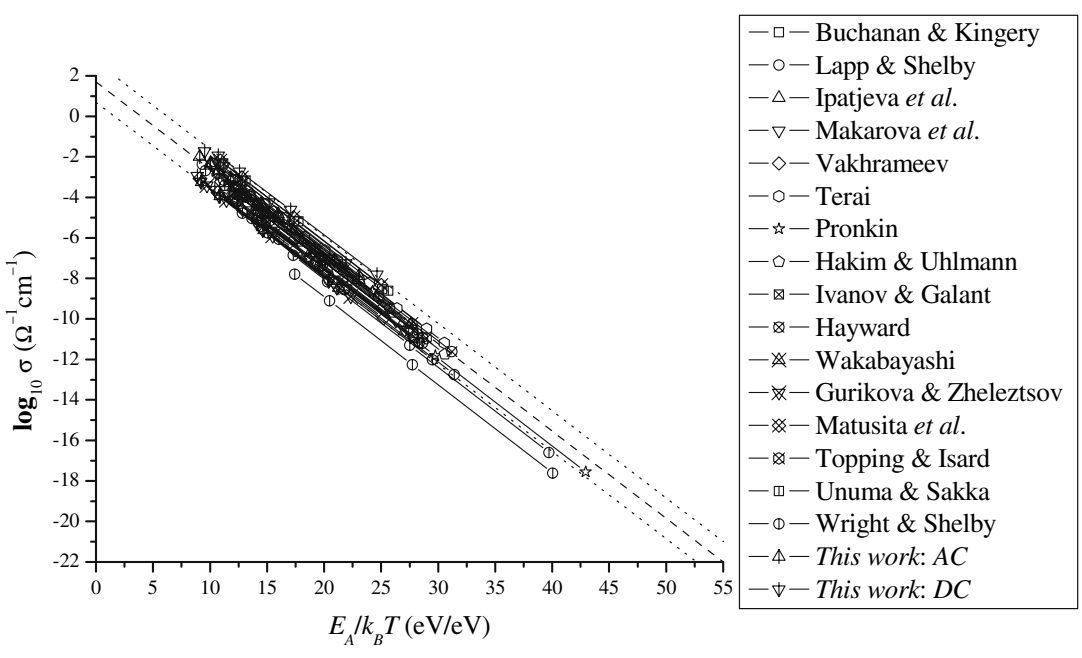

and germanate glasses, according to Eqs. 1 and 7. This paper aimed to present new results considering just sodium silicate glasses (Fig. 6). From these studies, the influence of alkali content and temperature was minor on the pre-exponential terms, considering both expressions $\log _{10} \sigma$ and $\log _{10} \sigma T$.

Figure 6 shows modified Arrhenius plots of $\sigma$ for the 48 sodium silicate glasses, from $x=4$ to $45 \mathrm{~mol} \%$, as indicated), ranging from $2.2 \times 10^{-2} / \Omega \mathrm{cm}$ to $2.4 \times 10^{-18}$ / $\Omega \cdot \mathrm{cm}$ between $20^{\circ} \mathrm{C}$ and $400{ }^{\circ} \mathrm{C}$. The range of activation energy $E_{A}$ lies between 0.515 and $1.08 \mathrm{eV}$ in all glasses studied, as indicated in Fig. 5. These data were compared with the 'universal' equation for $\sigma_{0}=50 /$ $\Omega \mathrm{cm}$ in Eq. 1. Following previous work by Nascimento et al., this "universal" equation appears in Fig. 6 as a dashed line, and the dotted lines are the highest and lowest limits within one magnitude order. Only one composition does not follow the 'universal' finding, which that from Wrigth and Shelby data [25] $\left(4 \mathrm{Na}_{2} \mathrm{O}\right.$ mol\%). It is important to note that this data correspond to the lowest limit of Fig. 5.

The replacement of a mobile ion with one of another type affects the ionic conductivity in various ways, such as causing modifications in the glass structure. But considering so many different binary sodium silicate glasses, as in Fig. 6, it is remarkable how strong is the correlation between $\sigma$ with $E_{A}$ and $T$.

The fact is that $\sigma$ lies on a single 'universal' curve in various sodium silicate glasses, whose conductivities differ by more than 16 orders of magnitude and within scatters of just one order of magnitude in $98 \%$ of the glass systems considered. It is also important to notice the wide composition range involved. Therefore, if one measures $\sigma$ at a fixed temperature, it is possible to estimate $E_{A}$ from Eq. 1 considering $\sigma_{0}=50 / \Omega \mathrm{cm}$, and obtain a rough sketch of $\sigma$ at different tempera- tures. This means that, if $E_{A}$ is obtained by some experimental or theoretical technique, the ionic conductivity can be readily calculated.

Another "universal" curve, following Eq. 7 and considering some binary silicate, borate and germanate glasses, resulted in the same 'universal' behavior [5254] as cited above. The pre-exponential value was $\sigma_{0}=50000 \mathrm{~K} / \Omega \mathrm{cm}$. The conclusions for this case also follows the above described considering Eq. 1. The most important fact is that, in Fig. 6, data for glasses of different compositions are unified by the single 'universal' Eq. 1 . The fact that $\sigma$ lies on this single 'universal' curve for many ion-conducting glasses means that $\sigma$ is mainly governed by $E_{A}$.

Therefore, Fig. 6 shows a 'universal curve' plotting conductivities and activation energies. In order to investigate the possibility of another general relation between ionic conductivity and the volume occupied by the network skeleton, the author calculated the expansion $\left(V_{m}-V\right) / V$ of the network, where $V$ and $V_{m}$ are the calculated network volume of $(1-x) \mathrm{SiO}_{2}$ forming unities (where $x$ corresponds to $\mathrm{Na}_{2} \mathrm{O}$ increment in $\mathrm{mol} \%$ ) and the experimental molar volume, respectively. Conductivity and molar volume data were considered from the same batches in Figs. 1-2, respectively. As shown in Fig. 2, the dopant $\mathrm{Na}_{2} \mathrm{O}$ added decreases the experimental molar volume before occupied by $\mathrm{SiO}_{2}$. The volume of pure silica was assumed as $27.232 \mathrm{~cm}^{3} / \mathrm{mol}$. The difference $V_{m}-V$ increases slightly and could be considered as proportional to the free volume, following similar procedure done by Swenson and Börjesson [47]. This is a rather rough approximation: the increase in molar volume of $\mathrm{Na}_{2} \mathrm{O}$ unities is the main factor involved in the increasing in conductivity and also in the free volume. Thus, the free volume defined here is a macroscopic quantity. The necessary condition for ion 
Fig. 7 Ionic conductivity $\sigma$ measured at $20^{\circ} \mathrm{C}$ versus the cubic root of expansion of the glass network $F$ estimated from density measurements at the same temperature. The line is just a guide for eye

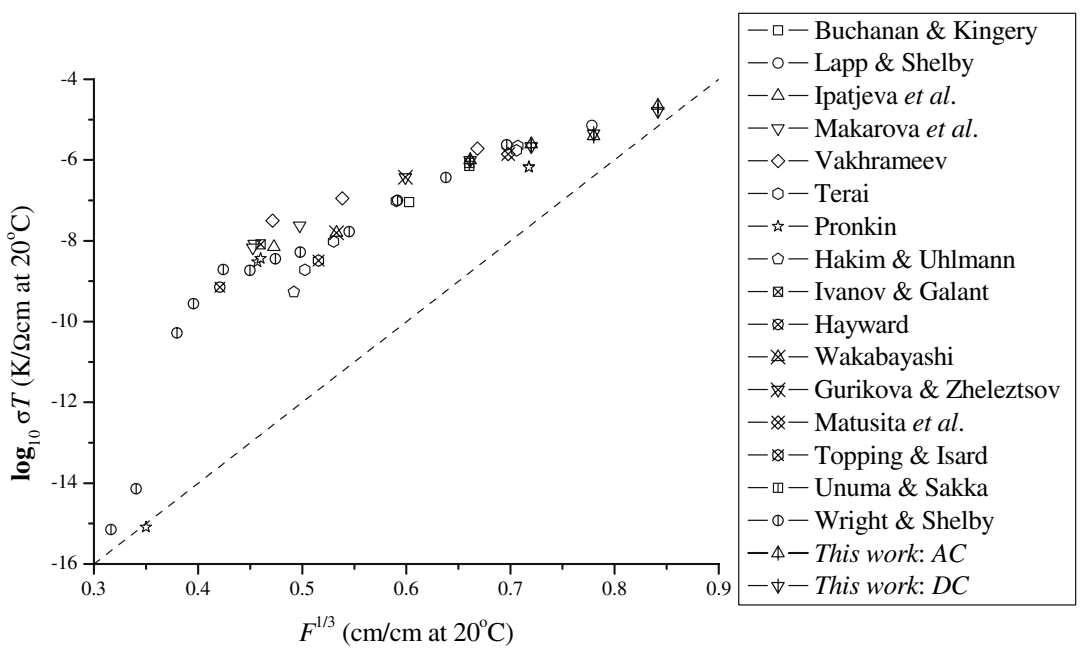

transport may rather be the presence of microscopic pathways available for sodium ions. A given material may be called 'conductive' if it is equipped with ample ionic pathways, irrespective of the amount of the free volume. Better approximation of free volume could be provided using positron annihilation spectroscopy, as recently published [55].

The glass systems in Fig. 7 cover a wide composition range, between 4 and $45 \mathrm{~mol} \%$. A striking common relation between the conductivity at $20{ }^{\circ} \mathrm{C}$ and the cubic root of free volume $F=\left(V_{m}-V\right) / V$ calculated from density of the same batches and at same temperature is evident; i.e., for a given expansion, all the different systems respond with the same increase on $\sigma$, regardless chemical (such as relative water content) or microstructural details (such as phase separation). Note that data in Fig. 7 represent $\sigma T$ values that vary by 10 orders of magnitude. The relation found is not linear, what could mean that the conductivity (or $\sigma T$ ) is more dependent on the number of mobile ions than the on free volume itself.

Fig. 8 Correlation between the experimental activation energy for ionic conductivity $E_{A}$ and the volume fraction $F$ estimated from density measurements considering a fixed temperature of $20^{\circ} \mathrm{C}$. The line is just a guide for eye
The common behavior of the conductivity increase with expansion of the network structure observed for the various binary sodium glasses suggests that the excess volume introduced by the dopant is an important parameter that influences the conductivity properties, as expressed by Eq. 7, but is not the key factor. Thus, at first sight it appears that the details of the microscopic structure have direct impact on the ionic conductivity in this system. For example, it should be noted that the microscopic interactions (mainly mechanical and dielectrical, as predicted by the Anderson-Stuart theory) lead to variations of the degree of expansion. For this reason, in order to explain the conducting properties and the increase the ionic conductivity with alkali content we focused on the A-S theory.

It is interesting to note that the increase in ionic conductivity is almost entirely due to the fact that the activation energy $E_{A}$ required for a cation jump decreases, as presented in Fig. 5. Thus, the pre-exponential term in

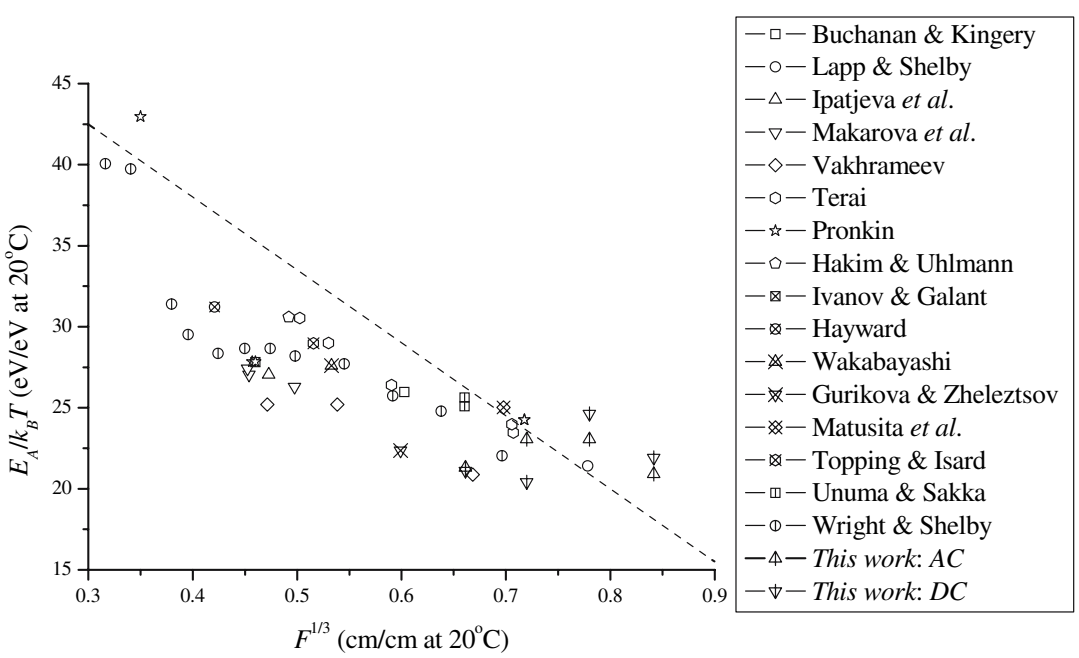


the Arrhenius law in Eq. 1, $\sigma_{0}$, is unaffected by alkali addition, as shown in Fig. 6.

The present finding on the common scaling between the conductivity enhancement and the expansion suggests that the expansion of the glass skeleton and therefore the strain energy part $E_{s}$ influence on the conduction properties in this system, as presented in Fig. 6.

Figure 8 shows that an increase in volume fraction reduces the activation energy for an ionic jump, which demonstrates that $E_{A} / k_{B} T$ varies roughly with the cube root of the volume fraction $F$. Thus, this approach, besides not linear, emphasizes the importance of "free volume" to the ion mobility.

\section{Conclusions}

The Anderson-Stuart model (A-S) has been used for a soda-silica glass system to describe the variation of the activation energy against composition in a wide composition range (from 4 up to $45 \mathrm{Na}_{2} \mathrm{O}$ mole\%), with reasonable results. Theoretical activation energy $E_{A}$ of sodium silicate glasses seems to vary smoothly with composition. For the first time considerations on experimental shear modulus $G$ and relative dielectric permittivity $\varepsilon$ with sodium composition fit well experimental available data. Shear stress has given more influence on fitting than relative dielectric permittivity. Considering the A-S theory, sodium silicate conducting glasses fall into an identifiable pattern where conductivity is related to chemical composition. A 'universal' finding is obtained using $\log _{10} \sigma$ vs. $E_{A} / k_{B} T$ in this binary silicate system. As a consequence, the pre-exponential factor of the conductivity $\sigma_{0}$ is composition independent. Another simple relation between the increase in ionic conductivity and the expansion of the glass network skeleton is revealed for very different binary sodium silicate glasses. The results show that an open structure with excess free volume, in addition to the alkali content, plays a significant role in promoting ionic conductivity. One should point that that the true free volume (from theory) is limited in all glasses, irrespective of the network modifier or dopant concentration. Therefore, the approach considered here was to relate ionic conductivity with the "expansion of the glass network" (or the "free volume") originated from the conduction pathways.

Acknowledgements The author acknowledges the Brazilian agencies RHAE, FAPESP and CNPq for the grant, Drs. E. D. Zanotto and G. P. Souza (UFSCar, Brazil) and Dr. V. M. Fokin (Vavilov State Optical Institute, Russia) for valuable sugges- tions. Special thanks to Dr. R. Muccillo (IPEN, Brazil) for measurements of $A C$ conductivities and helpful assistance.

\section{References}

1. Angell CA (1992) Ann Rev Phys Chem 43:693

2. Ravaine D, Souquet JL (1977) Phys Chem Glasses 18:27

3. Funke K (1993) Prog Solid State Chem 22:11

4. Anderson OL, Stuart DA (1954) J Am Ceram Soc 37:573

5. Bunde A, Ingram MD, Maass P (1994) J Non-Cryst Solids 172/174:1222

6. Hakim RM, Uhlmann DR (1971) Phys Chem Glasses 12:132

7. Nascimento MLF (2000) MSc Dissertation. Institute of Physics, University of São Paulo (in Portuguese)

8. Buchanan RC, Kingery WD (1965) Compt Rend VII Congr Intern du Verre Bruxelles 2:368

9. Lapp JC, Shelby JE (1986) J Non-Cryst Solids 84:463

10. Ipatjeva VV, Borisova ZU, Molchanov VS (1967) Zh Prikl Khim 40:1424

11. Makarova TM, Molchanov VS (1961) Opt Mekh Prom 2:26

12. Makarova TM, Mazurin OV, Molchanov VS (1960) Izv Vyssh Uchebn Zaved Khimiya i Khim Tekhnol 3:1072

13. Vakhrameev VI (1968) Steklo 3:84

14. Terai R (1969) J Ceram Soc Jpn 77:318

15. Pronkin AA (1965) In: Khimiya Tverdogo Tela, Leningrad 125

16. Pronkin AA (1979) Fizika i Khimiya Stekla 5:634

17. Hakim RM, Uhlmann DR (1967) Phys Chem Glasses 8:174

18. Ivanov AO, Galant EI (1963) Opt Mekh Prom 3:43

19. Hayward PJ (1976) Phys Chem Glasses 17:54

20. Wakabayashi H (1989) Phys Chem Glasses 30:51

21. Gurikova LM, Zheleztsov VA (1976) Steklo 1:15

22. Matusita K, Takayama S, Sakka S (1980) J Non-Cryst Solids 40:149

23. Topping JA, Isard JO (1971) Phys Chem Glasses 12:145

24. Unuma H, Sakka S (1987) J Mater Sci Lett 6:996

25. Wright BM, Shelby JE (2000) Phys Chem Glasses 41:192

26. Haller W, Blackburn DR, Simmons JH (1974) J Am Ceram Soc $57: 120$

27. Martin SW, Angell CA (1986) J Non-Cryst Solids 83:185

28. McElfresh DK, Howitt DG (1986) J Am Ceram Soc 69:C237

29. Appen AA, Kozlovskaya EI, Fuxi G (1961) Zh Prikhl Khim 34:975

30. Eagan RJ, Swearengen JC (1978) J Am Ceram Soc 61:27

31. Halleck PM, Pacalo RE, Graham EK (1986) J Non-Cryst Solids 86:190

32. Karapetian GO, Livshits VY, Tennisson DG (1981) Fiz Khim Stekla 7:188

33. Livshits VY, Tennisson DG, Gukasyan SB, Kostanyan KA (1982) Fiz Khim Stekla 8:688

34. Manghnani MH, Singh BK (1974) Proc Xth Inter Cong Glass, Kyoto 104

35. Molot VA (1992) MSc Thesis

36. Takahashi K, Osaka A (1983) J Ceram Soc Jpn 91:116

37. DG Tennison, PhD Thesis, Leningrad (1981)

38. Matusita K, Sakka S, Osaka K, Soga N, Kunugi M (1974) J Non-Cryst Solids 16:308

39. Appen AA, Fuxi G (1959) Fizika Tverdogo Tela 1:1529

40. Keller F (1932) Z Techn Physik 13:237

41. Stockdale GF (1953) Univ III Bull 50:411

42. Taylor HE (1956) Trans Faraday Soc 52:873

43. Unuma H, Sakka S (1987) J Mater Sci Lett 6:996

44. Takata M, Tomozawa M, Watson EB (1982) J Am Ceram Soc 65:91 
45. Nascimento MLF, do Nascimento E, Pontuschka WM, Matusoka M, Watanabe S (2006) Ceramica 52:22

46. Nascimento MLF, do Nascimento E, Watanabe S (2005) Braz J Phys 35:626

47. Swenson J, Börjesson L (1996) Phys Rev Lett 77:3569

48. Adams S, Swenson J (2002) Phys Chem Chem Phys 4:3179

49. Swenson J, Adams S (2002) Phys Rev B 64:024204

50. Adams S, Swenson J (2002) Solid State Ion 154/155:151
51. Adams S, Swenson J (2000) Phys Rev Lett 84:4144

52. Nascimento MLF, Watanabe S (2005) J Mat Sci 40:5079

53. Nascimento MLF, Watanabe S (2005) J Mat Sci 40:4423

54. Nascimento MLF, do Nascimento E, Watanabe S (2006) Mat Chem Phys 96:55

55. Ingram MD, Pas SJ, Cramer C, Gao Y, Hill AJ (2005) Phys Chem Chem Phys 7:1620 